\title{
Maximum black-hole spin from quasi-circular binary mergers
}

\author{
Michael Kesden* \\ Center for Cosmology and Particle Physics, New York University, \\ Department of Physics, 4 Washington Pl., New York, New York 10003 \\ Guglielmo Lockhart and E. Sterl Phinney ${ }^{\dagger}$ \\ California Institute of Technology, MC 350-17, 1216 E. California Blvd., Pasadena, California 91125
}

(Dated: October 2010)

\begin{abstract}
Black holes of mass $M$ must have a spin angular momentum $S$ below the Kerr limit $\left(\chi \equiv S / M^{2} \leq\right.$ 1), but whether astrophysical black holes can attain this limiting spin depends on their accretion history. Gas accretion from a thin disk limits the black-hole spin to $\chi_{\text {gas }} \lesssim 0.9980 \pm 0.0002$, as electromagnetic radiation from this disk with retrograde angular momentum is preferentially absorbed by the black hole. Extrapolation of numerical-relativity simulations of equal-mass binary black-hole mergers to maximum initial spins suggests these mergers yield a maximum spin $\chi_{e q} \lesssim 0.95$. Here we show that for smaller mass ratios $q \equiv m / M \ll 1$, the superradiant extraction of angular momentum from the larger black hole imposes a fundamental limit $\chi_{\text {lim }} \lesssim 0.9979 \pm 0.0001$ on the final black-hole spin even in the test-particle limit $(q \rightarrow 0)$ of binary black-hole mergers. The nearly equal values of $\chi_{\text {gas }}$ and $\chi_{\text {lim }}$ imply that measurement of supermassive black-hole spins cannot distinguish a black hole built by gas accretion from one assembled by the gravitational inspiral of a disk of compact stellar remnants. We also show how superradiant scattering alters the mass and spin predicted by models derived from extrapolating test-particle mergers to finite mass ratios.
\end{abstract}

\section{INTRODUCTION}

Supermassive black holes (SBHs) reside in the centers of most large galaxies. While a few nearby SBHs can be detected by their gravitational influence on surrounding stars, the majority of SBHs are observed electromagnetically as active galactic nuclei (AGN). The same accretion flows that release energy to power AGN also supply energy and angular momentum to the black holes themselves increasing their mass $M$ and spin $S$ [43]. By definition, black holes possess an event horizon from within which nothing can escape to future null infinity. Black holes described by the Kerr metric [1] only possess an event horizon for $\chi \equiv S / M^{2} \leq 1$, setting a fundamental upper limit on a black hole's possible spin. Whether or not astrophysical SBHs saturate or even exceed this Kerr limit is an important test of general relativity.

Black-hole spins also probe their assembly history. SBHs grow both by gas accretion and mergers driven by the gravitational inspiral of binary companions. These two growth mechanisms may supply mass and angular momentum to the SBHs in different ratios, allowing measurements of black-hole spin to distinguish between them. Reverberation mapping of the iron $\mathrm{K} \alpha$ line in AGN x-ray spectra has been proposed as just such a means of measuring SBH spins [2]. XMM-Newton observations analyzed with this technique have been used to constrain the spin of the SBH hosted by the Seyfert 1.2 galaxy MCG-06-30-15 to $\chi=0.989_{-0.002}^{+0.009}$ at $90 \%$ confidence [3]. A closer examination of how SBHs acquire their spins is

*Electronic address: mhk10@nyu.edu

${ }^{\dagger}$ Electronic address: esp@tapir.caltech.edu thus of both theoretical and observational importance.

According to Bardeen [4, a nonspinning black hole can attain the maximum Kerr spin $\chi=1$ after accreting a finite mass of test particles freely falling from its innermost stable circular orbit (ISCO). However, material on circular orbits with radii greater than that of the ISCO cannot be accreted unless it has some mechanism to shed its excess angular momentum. In a standard geometrically thin, optically thick accretion disk [5, this mechanism is viscous stress within the disk that also heats it locally and transports energy outwards. This heating produces an energy flux at the disk's surface that will be radiated in all directions. A small fraction of these radiated photons do not escape to infinity but are instead captured by the black hole itself. Photons with negative angular momentum with respect to the black hole's spin have a larger capture cross-section than those with positive angular momentum [6], implying that the accreted radiation will counteract the directly advected material which always acts to spin the hole up for $\chi<1$. These two sources of angular momentum cancel for black-hole spins $\chi_{\text {gas }} \simeq 0.998$, with black holes of greater spins spinning down to this value after accreting a mass $m \simeq 0.05 M[7$.

Different accretion flows will supply energy and angular momentum to the black hole in different ratios, altering the value of the limiting spin $\chi_{\text {gas }}$. Advectiondominated accretion flows (ADAFs) do not cool efficiently, and therefore a fraction $f>0$ of the gravitational energy dissipated prior to the ISCO is advected by the black hole rather than radiated to infinity. Viscous stresses in these flows proportional to the ShakuraSunyaev parameter $\alpha$ [5] also reduce the specific angular momentum of the accreted material below its value in the vacuum at the ISCO. The former effect increases $M$ in the denominator in the definition $\chi \equiv S / M^{2}$ of the di- 
mensionless spin, while the latter effect reduces $S$ in the numerator [8]. The magnetorotational instability 9 also torques gas at the ISCO, and in addition can launch jets which further limit the spin as shown in magnetohydrodynamic simulations [10]. Analytic fits to the ADAF simulations of Popham and Gammie [8] suggest that black holes can spin up to $\chi_{\mathrm{ADAF}} \simeq 0.96$, and that the inclusion of jets calibrated by the magnetohydrodynamic simulations of Gammie et al. [10] reduces this limit to $\chi_{\text {jets }} \simeq 0.93[11$. We see that limits on black-hole spin depend greatly on the nature of the accretion flow, and that no model-independent constraints exclude the limit $\chi_{\text {gas }} \simeq 0.998$ for thin disks first obtained by Thorne [7.

The maximum spin of black holes produced in binary mergers is also greatly uncertain. The most accurate numerical-relativity simulations have been of equal-mass $q \equiv m_{2} / m_{1}=1$ black holes with spins $\chi_{1}=\chi_{2}$ aligned or antialigned with the orbital angular momentum. These simulations found $\chi=0.68646 \pm 0.00004$ for nonspinning binaries [12], and $\chi=0.547812 \pm 0.000009$ for equalmass binaries with initial spins $\chi_{1}=\chi_{2}=-0.43757[13$. The spins of such aligned binaries do not precess prior to merger, removing one complication in determining their magnitude and direction. We will therefore restrict our analysis to aligned binaries in this paper, though with modest additional work it could be extended to binaries of arbitrarily oriented spins.

Although these numerically determined spins are remarkably precise, two significant obstacles still prevent the determination of final black-hole spins from more generic mergers. The first is the inability of the commonly used conformally flat Bowen-York initial data to adequately approximate initial binary black holes with spins greater than $\chi_{i} \simeq 0.93$ [14]. An extrapolation of simulations with $\chi_{i} \leq 0.9$ suggests that the merger of equal-mass black holes with maximal initial spins aligned with their orbital angular momentum yields a final black hole with spin $\chi=0.951 \pm 0.004$ [15. However, it remains to be proven that such a smooth extrapolation in initial spin holds all the way to the Kerr limit.

The second obstacle to determining spins from generic mergers is the increased computational resources needed to simulate mergers with mass ratios $q \leq 1$. This increased computational demand results from the need for more closely spaced numerical grid points to resolve the horizon of the smaller black hole, shorter timesteps owing to the shorter light propagation time between grid points, and longer simulations to capture the larger number of orbits per unit increase in orbital frequency prior to merger. The smallest mass ratio that has currently been simulated is $q=0.01$ [16]; only a handful of simulated mergers with $q \leq 0.1$, all of initially nonspinning black holes, have been published [17 19]. New numerical techniques will be required to make much progress beyond this point.

Relativists have attempted to surmount these obstacles by inventing fitting formulas that are functions of $q, \chi_{1}$, and $\chi_{2}$, calibrating the coefficients in these for- mulas with existing simulations, and then extrapolating them to higher spins and lower mass ratios than can currently be simulated. This approach is very effective in the region of the parameter space $\left\{q, \chi_{1}, \chi_{2}\right\}$ near the simulations with which the fitting formulas were calibrated, but can break down outside this region. The probable reason for this is that all three parameters listed above equal unity for equal-mass, maximally spinning, aligned mergers, and thus polynomials in these parameters will converge slowly if at all. Even the symmetric mass ratio $\eta \equiv m_{1} m_{2} /\left(m_{1}+m_{2}\right)^{2}=q /(1+q)^{2}$ gets as large as $1 / 4$ for equal-mass mergers, suggesting that polynomials in this parameter will not converge quickly either. At the time this paper was written, simulations were restricted to the region $0.1 \leq q \leq 1(0.0826 \leq \eta \leq 0.25)$ which provided a short lever arm over which to calibrate terms with different $q$ dependence. The degeneracy between terms makes predicting final spins in the test-particle $(q \rightarrow 0)$ limit very uncertain. For example, several fitting formulas predict final spins above the Kerr limit for maximally spinning aligned mergers with mass ratios as modest as $q \lesssim 0.25$. The publication of a simulation with $q=0.01$ [16] during the preparation of this paper shows the rapid progress towards numerical simulations of extreme-massratio mergers, but much work remains before such simulations are available for generically spinning black holes.

Fortunately our analytical knowledge of the geodesics of the Kerr metric can help us understand binary blackhole mergers in the test-particle limit. These mergers can be broken down into three stages: inspiral, plunge, and ringdown. During the inspiral stage, the orbit of the test particle adiabatically evolves through a series of geodesics with successively lower energy and angular momentum as these quantities are radiated away through the emission of gravitational waves. This gravitational radiation circularizes initially eccentric orbits while the evolution is post-Newtonian [20], implying that for many though possibly not all astrophysical mergers the orbit will have circularized before the test particle reaches the ISCO. After this point, during the brief plunge stage, the test particle rapidly falls into the event horizon of the larger black hole. Finally, the quasinormal modes excited during the merger ring down as the newly formed black hole settles into its final Kerr configuration. In the test-particle limit, the energy and angular momentum radiated during the inspiral stage scales linearly with $q$, while that radiated during plunge and ringdown scales as a higher power in the small parameter $q$. This suggests that one can reasonably predict the mass and spin of the final black hole by equating these quantities to the energy and total angular momentum of the binary at the ISCO.

This prediction assumes that during the inspiral all of the gravitational waves are radiated outwards to infinity rather than downwards to the larger black hole's event horizon. For highly spinning black holes, these downward gravitational waves will be superradiantly scattered, extracting energy and angular momentum from the larger black hole. Individual modes can be amplified as much as 
$138 \%$ by a maximally spinning black hole [21]; the total energy flux radiated to infinity by a test particle at the ISCO will be amplified by $12.9 \%$ for a black hole of spin $\chi=0.999$ [22]. Although this amplification decreases rapidly as a function of the orbital radius, the total energy and angular momentum extracted throughout the inspiral still scale linearly in $q$ and will therefore remain the dominant factor affecting the final mass and spin after the energy and angular momentum advected with the test particle itself during merger.

The primary goal of this paper is to determine how much the superradiant scattering of gravitational waves during the inspiral reduces the maximum spin achievable by binary black-hole mergers below the Kerr limit first predicted by Bardeen [4. In Sec. II we will review the previous analytic predictions for the final mass and spin that serve as our starting point. Our method for calculating the energy and angular momentum extracted from the spinning black hole will be described in Sec. III. The results of this calculation are presented in Sec. IV] In Sec. V we compare our model to others in the literature, extrapolate it to comparable-mass mergers and compare with numerical-relativity simulations, and suggest how it might be combined with fitting formulas to produce an approximation valid over the entire domain $0 \leq q \leq 1$. A brief summary and a few final remarks on the astrophysical implications of our analysis are offered in Sec. [VI]

\section{TEST-PARTICLE MERGERS}

Hughes and Blandford [23] (hereafter HB) recognized that in the test-particle limit, the energy and angular momentum radiated during plunge and ringdown was much less than that released during the inspiral stage. They predicted the mass $M_{f}$ and spin $S_{f}$ of the final black hole would therefore be given by

$$
\begin{aligned}
M_{f, \mathrm{HB}} & =m_{1}+m_{2} E_{\mathrm{ISCO}}\left(\chi_{1}\right), \\
S_{f, \mathrm{HB}} & =m_{1} m_{2} L_{\mathrm{ISCO}}\left(\chi_{1}\right)+m_{1}^{2} \chi_{1} .
\end{aligned}
$$

Here, $E_{\mathrm{ISCO}}(\chi)$ is the energy per unit mass of a test particle on the equatorial ISCO of a Kerr black hole with dimensionless spin $\chi . L_{\mathrm{ISCO}}(\chi)$ is the corresponding dimensionless orbital angular momentum. The dimensionless final spin is simply $\chi_{f}=S_{f} / M_{f}^{2}$. This formula is exact in the test-particle limit, and for $q \rightarrow 0, \chi_{1} \rightarrow 1$ correctly reproduces the Bardeen result

$$
\frac{\partial \chi_{f, \mathrm{HB}}}{\partial q} \rightarrow L_{\mathrm{ISCO}}(1)-2 E_{\mathrm{ISCO}}(1)=0
$$

Maximally spinning black holes cannot be spun up above the Kerr limit.

Although Eqs. (1) are exact in the test-particle limit, they are manifestly asymmetric in the black-hole labels "1" and "2". For example, they ignore altogether the spin $\chi_{2}$ of the smaller black hole. While HB boldly extrapolate their formula to $q=0.5$, Buonanno, Kidder, and
Lehner 24] (hereafter BKL) realized that a symmetrized version would more accurately describe comparable-mass mergers. They proposed

$$
\begin{aligned}
M_{f, \mathrm{BKL}} & =m_{1}+m_{2}, \\
S_{f, \mathrm{BKL}} & =m_{1} m_{2} L_{\mathrm{ISCO}}\left(\chi_{f}\right)+m_{1}^{2} \chi_{1}+m_{2}^{2} \chi_{2} .
\end{aligned}
$$

In addition to the obvious improvement of including the second spin $\chi_{2}$, BKL made the inspired choice of using the dimensionless spin $\chi_{f}$ of the final black hole to calculate the orbital angular momentum of the initial binary at the ISCO. Although counterintuitive at first, this choice correctly captures the total energy and angular momentum of the system which are assumed to be conserved after the binary reaches the ISCO.

Equations. (3) are far more successful at predicting the final spin from equal-mass mergers than they have any right to be. They predict that equal-mass nonspinning black holes will merge to yield a final black hole with spin $\chi_{f, \mathrm{BKL}}=0.663$ quite close to the numerically determined value $\chi_{\mathrm{NR}}=0.68646 \pm 0.00004$ [12]. Equal-mass, maximally spinning black holes are predicted to produce a final spin $\chi_{f, \mathrm{BKL}}=0.959$ which is also surprisingly close the numerically extrapolated value $\chi_{\mathrm{NR}}=0.951 \pm 0.004$ [15]. This latter agreement however is an artifact of overestimating the final mass in Eq. (3a) by failing to account for the energy radiated during the inspiral. Overestimating the denominator in the expression $\chi_{f}=S_{f} / M_{f}^{2}$ leads to an underestimate of the final spin. In the limit $q \rightarrow 0, \chi_{1} \rightarrow 1$, this underestimation implies

$$
\frac{\partial \chi_{f, \mathrm{BKL}}}{\partial q} \rightarrow L_{\mathrm{ISCO}}(1)-2=2\left(3^{-1 / 2}-1\right)<0
$$

Maximally spinning black holes are artificially found to be spun down by test-particle mergers. In this model black holes can only be spun up by test particles to the fictitious limit $\chi_{\mathrm{lim}}=0.948$ at which $\partial \chi_{f} / \partial q=0$.

Kesden 25. (hereafter $\mathrm{K}$ ) sought to remedy this by replacing Eq. 3a with

$$
M_{f, \mathrm{~K}}=M-\mu\left[1-E_{\mathrm{ISCO}}\left(\chi_{f}\right)\right],
$$

where $M \equiv m_{1}+m_{2}$ is the sum of the initial masses, $\mu \equiv m_{1} m_{2} / M$ is the reduced mass of the binary, and the energy per unit mass $E_{\mathrm{ISCO}}\left(\chi_{f}\right)$ is evaluated using the final dimensionless spin in the spirit of BKL. This formula retains the desired symmetry of Eqs. (3) under exchange of black-hole labels, but also reduces to Eqs. (1) in the test-particle limit thereby preserving the Bardeen result that black holes can be spun all the way up to the Kerr limit by test-particle mergers. It predicts that equalmass, nonspinning black holes will merge into a hole with final spin $\chi_{f, \mathrm{~K}, \mathrm{NS}}=0.687$ in near miraculous (and probably coincidental) agreement with numerical simulations, but also predicts an uncomfortably large final spin $\chi_{f, \mathrm{~K}, \mathrm{~S}}=0.9988$ for maximally spinning aligned mergers. Equations (3b) and (5) are not the unique choice that possesses the desired symmetry and limiting behavior. 
For example, replacing Eq. 3b with

$$
S_{f, \mathrm{~K}}=\mu M_{f} L_{\mathrm{ISCO}}\left(\chi_{f}\right)+m_{1}^{2} \chi_{1}+m_{2}^{2} \chi_{2}
$$

also maintains these properties, but predicts different results $\chi_{f, \mathrm{~K}, \mathrm{NS}}=0.675$ and $\chi_{f, \mathrm{~K}, \mathrm{~S}}=0.9909$ when extrapolated to equal-mass mergers. For our purpose, in this paper of calculating the maximum final spin for testparticle mergers, either Eqs. (1a) or Eq. (5) paired with either Eq. (3b) or Eq. (6) can serve as a suitable starting point.

\section{SUPERRADIANT SCATTERING}

The gravitational radiation emitted by an inspiraling test particle is fully described by the complex Weyl scalar

$$
\psi_{4} \equiv-C_{\alpha \beta \gamma \delta} n^{\alpha} \bar{m}^{\beta} n^{\gamma} \bar{m}^{\delta},
$$

where $C_{\alpha \beta \gamma \delta}$ is the Weyl curvature tensor and $n^{\mu}$ and $\bar{m}^{\mu}$ are elements of a Newman-Penrose tetrad of null 4vectors [26. Teukolsky [27] decomposed $\psi_{4}$ into multipole moments

$\psi_{4}=(r-i a \cos \theta)^{-4} \int_{-\infty}^{\infty} d \omega \sum_{l m} R_{l m \omega}(r)_{-2} S_{l m}^{a \omega}(\theta) e^{i m \phi} e^{-i \omega t}$

and showed that the evolution of the radial modes $R_{l m \omega}(r)$ is governed by an ordinary second-order differential equation sourced by the test particle's contribution to the stress-energy tensor. Sasaki and Nakamura 28 derived a linear transformation of $R_{l m \omega}(r)$ that greatly facilitates the solution of this differential equation. To solve this equation ourselves, we used the GREMLIN (Gravitational Radiation in the Extreme Mass ratio LIMit) code written and generously provided to us by Scott Hughes. We adopt his notation and closely follow his treatment presented in 29] to calculate the energy and angular momentum extracted from the black hole by superradiant scattering during the inspiral.

The Teukolsky-Sasaki-Nakamura formalism allows one to calculate the rates $(d E / d t)_{r \rightarrow r_{+}}^{\mathrm{rad}}$ and $\left(d L_{z} / d t\right)_{r \rightarrow r_{+}}^{\mathrm{rad}}$ at which energy and angular momentum are radiated down the event horizon at $r_{+}$. The total energy $E_{\mathrm{SR}}$ and angular momentum $J_{\mathrm{SR}}$ extracted from the black hole as the test particle inspirals from infinity to $r$ are integrals of these fluxes

$$
\begin{aligned}
& E_{\mathrm{SR}}(\chi, r)=\int_{r}^{\infty}\left(\frac{d E}{d t}\right)_{r \rightarrow r_{+}}^{\mathrm{rad}} \frac{d r^{\prime}}{\dot{r}}, \\
& J_{\mathrm{SR}}(\chi, r)=\int_{r}^{\infty}\left(\frac{d L_{z}}{d t}\right)_{r \rightarrow r_{+}}^{\mathrm{rad}} \frac{d r^{\prime}}{\dot{r}},
\end{aligned}
$$

where the radial velocity $\dot{r}$ during an adiabatic, quasicircular inspiral is given by

$$
\dot{r}(\chi, r)=\left(\frac{d E}{d t}\right)_{\mathrm{tot}}^{\mathrm{rad}}\left(\frac{d E}{d r}\right)^{-1} .
$$

Since $\dot{r}$ is negative as are the fluxes $(d E / d t)_{r \rightarrow r_{+}}^{\mathrm{rad}}$ and $\left(d L_{z} / d t\right)_{r \rightarrow r_{+}}^{\mathrm{rad}}$ for high spins, $E_{\mathrm{SR}}$ and $J_{\mathrm{SR}}$ are defined to be positive when energy and angular momentum are extracted from the black hole and negative when they are absorbed. Adding the contributions of Eqs. (9) to the right-hand sides of Equations (5) and (3b) yields

$$
\begin{aligned}
M_{f}= & M-\mu\left[1-E_{\mathrm{ISCO}}\left(\chi_{f}\right)+E_{\mathrm{SR}}\left(\chi_{f}, r_{\mathrm{ISCO}}\right)\right] \\
S_{f}= & m_{1} m_{2}\left[L_{\mathrm{ISCO}}\left(\chi_{f}\right)-J_{\mathrm{SR}}\left(\chi_{f}, r_{\mathrm{ISCO}}\right)\right] \\
& \quad+m_{1}^{2} \chi_{1}+m_{2}^{2} \chi_{2} \\
\chi_{f}= & S_{f} / M_{f}^{2}
\end{aligned}
$$

for our revised prediction for the final black-hole mass and spin. With a slightly different extrapolation of $S_{f}$ to large mass ratios, we can modify Eq. (6) to obtain

$$
\begin{gathered}
S_{f}^{\prime}=\mu M_{f}\left[L_{\mathrm{ISCO}}\left(\chi_{f}\right)-J_{\mathrm{SR}}\left(\chi_{f}, r_{\mathrm{ISCO}}\right)\right] \\
+m_{1}^{2} \chi_{1}+m_{2}^{2} \chi_{2} .
\end{gathered}
$$

In the test-particle limit $q \rightarrow 0$, Eq. (11a) combined with either Eq. (11b) or Eq. (12) gives

$$
\begin{aligned}
\frac{\partial \chi_{f}}{\partial q}\left(\chi_{1}, r\right) & \equiv \frac{\partial \chi_{\mathrm{ISCO}}}{\partial q}\left(\chi_{1}\right)-\frac{\partial \chi_{\mathrm{SR}}}{\partial q}\left(\chi_{1}, r\right) \\
\frac{\partial \chi_{\mathrm{ISCO}}}{\partial q}\left(\chi_{1}\right) & \rightarrow L_{\mathrm{ISCO}}\left(\chi_{1}\right)-2 \chi_{1} E_{\mathrm{ISCO}}\left(\chi_{1}\right) \\
\frac{\partial \chi_{\mathrm{SR}}}{\partial q}\left(\chi_{1}, r\right) & \rightarrow J_{\mathrm{SR}}\left(\chi_{1}, r\right)-2 \chi_{1} E_{\mathrm{SR}}\left(\chi_{1}, r\right) .
\end{aligned}
$$

At the limiting spin $\chi_{1}=\chi_{\lim }, \partial \chi_{f}\left(\chi_{1}, r_{\mathrm{ISCO}}\right) / \partial q=0$, implying that black holes cannot be spun up beyond $\chi_{\text {lim }}$ by test-particle mergers.

\section{RESULTS}

In the top panel of Fig. 1, we show the dimensionless energy $E_{\mathrm{SR}}(\chi, r)$ and angular momentum $J_{\mathrm{SR}}(\chi, r)$ extracted from a black hole of spin $\chi_{\mathrm{lim}}=0.9979$ by superradiant scattering as a test particle inspirals from infinity to a radius $r$. According to Eq. (13c), these two quantities determine the spin decrease $\partial \chi_{\mathrm{SR}} / \partial q$ due to the superradiant scattering of downward-going gravitational waves. This spin decrease is shown for spins $\chi=0.996$, 0.997, $\chi_{\lim }$, and 0.999 in the bottom panel of Fig. 1. The increase in spin $\partial \chi_{\mathrm{ISCO}} / \partial q$ when the test particle itself is accreted by black holes of the same spins is shown by dotted horizontal lines. At the limiting spin $\chi_{\mathrm{lim}}$, the total spin decrease $\partial \chi_{\mathrm{SR}} / \partial q$ evaluated at the ISCO precisely cancels the spin increase $\partial \chi_{\mathrm{ISCO}} / \partial q$ leaving the dimensionless spin $\chi$ unchanged. This is seen explicitly by the intersection of the solid black curve and dotted black line at $r_{\text {ISCO }}$ in the bottom panel of Fig. 1. The intersection of $\partial \chi_{\mathrm{SR}} / \partial q$ and $\partial \chi_{\mathrm{ISCO}} / \partial q$ at $\chi_{1}=\chi_{\mathrm{lim}}, r=r_{\mathrm{ISCO}}$ implies that the total spin change $\partial \chi_{f} / \partial q$ vanishes by Eq. (13a). Note that both the spin angular momentum $S_{f}$ and mass $M_{f}$ do increase as a result of the merger, but in just the right ratio as to preserve $\chi_{f}=S_{f} / M_{f}^{2}$. 


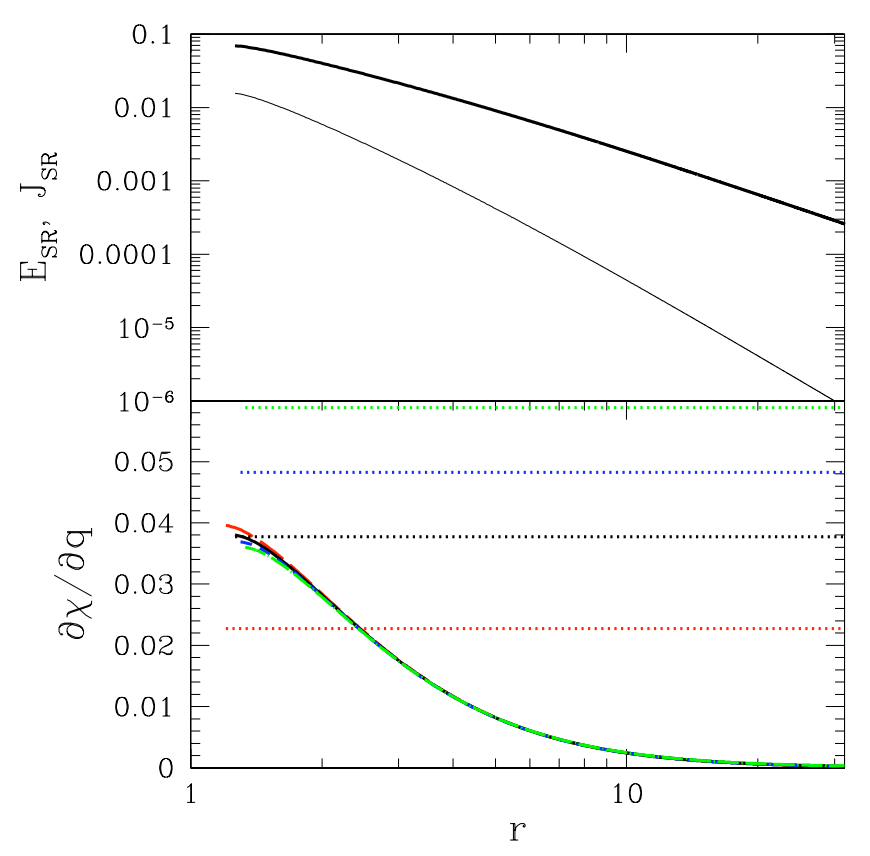

FIG. 1: Top panel: The dimensionless energy $E_{\mathrm{SR}}$ (thin line) and angular momentum $J_{\mathrm{SR}}$ (thick line) extracted from a black hole with the limiting spin $\chi_{\lim }=0.9979$ as the test particle inspirals from infinity to radius $r$. Bottom panel: The change in final spin per unit test-particle mass $\partial \chi / \partial q$. The dotted horizontal lines show the spin increase $\partial \chi_{\mathrm{ISCO}} / \partial q$ when the test particle falls from the ISCO into a black hole of spin $\chi=0.996$ (green), 0.997 (blue), $\chi_{\text {lim }}$ (black), and 0.999 (red). The curves $\partial \chi_{\mathrm{sR}} / \partial q$ show the spin decrease as superradiant scattering extracts energy from black holes with spins of the corresponding color. Only at $\chi=\chi_{\lim }$ do the lines and curves intersect at $r_{\mathrm{ISCO}}$, indicating that the spin remains unchanged by the merger.

The spin increase $\partial \chi_{\mathrm{ISCO}} / \partial q$ is a monotonically decreasing function of $\chi_{1}$, while the spin decrease $\partial \chi_{\mathrm{SR}} / \partial q$ evaluated at $r_{\text {ISCO }}$ monotonically increases with $\chi_{1}$. This implies that the total spin change $\partial \chi_{f} / \partial q$ monotonically decreases with $\chi_{1}$, intersecting zero at $\chi_{1}=\chi_{\text {lim }}$. Black holes with $\chi_{1}<\chi_{\lim }$ are spun up by mergers with test particles on circular equatorial orbits, while black holes with spins greater than $\chi_{\text {lim }}$ are conversely spun down. Black holes are never spun down $\left[\partial \chi_{f}\left(\chi_{1}, r_{\mathrm{ISCO}}\right) / \partial q \geq\right.$ $\left.0, \forall \chi_{1}\right]$ when superradiant scattering is neglected, reproducing the original Bardeen [4] result that the Kerr limit could be saturated.

Only one curve for $E_{\mathrm{SR}}$ and one for $J_{\mathrm{ISCO}}$ were presented in the top panel of Fig. 1. as the difference in these quantities as functions of the initial spin could not be distinguished in the logarithmic plot needed to depict the five orders-of magnitude of their evolution. In Fig. 2 , we zoom in on the region near the ISCO to display this spin dependence. The total energy and angular momentum extracted are nearly independent of spin all the way

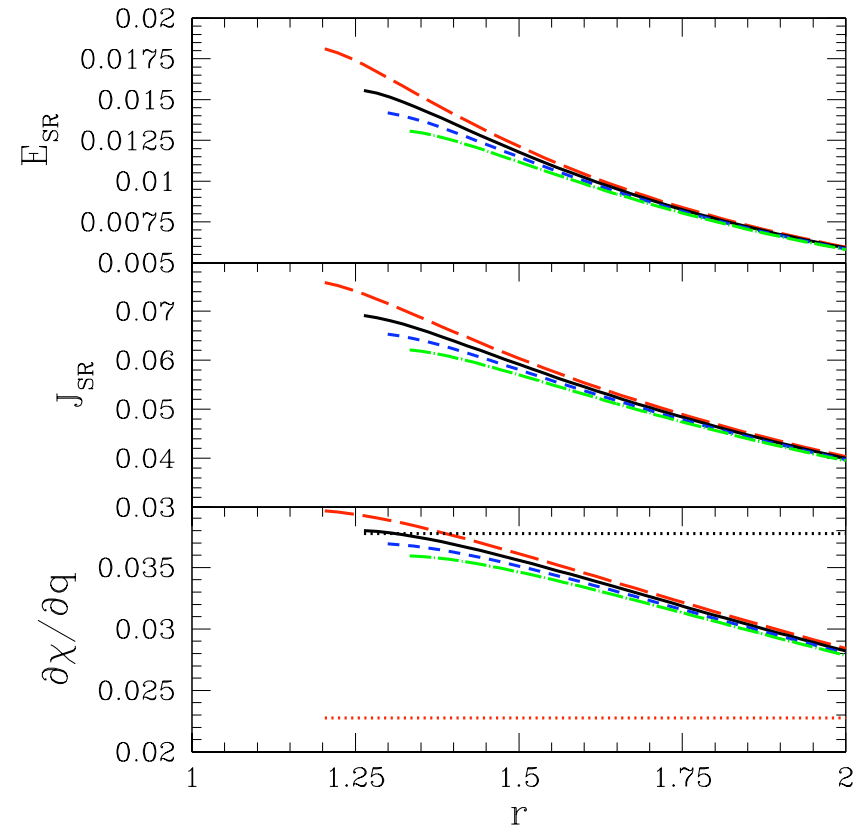

FIG. 2: The same quantities $E_{\mathrm{SR}}, J_{\mathrm{SR}}$, and $\partial \chi / \partial q$ presented in Fig. 1 as functions of the orbital radius $r$. We zoom into the region near the ISCO to better display how these quantities evolve near merger. The colors and styles of the curves are the same as those in Fig. 1 .

down to $r=2 m_{1}$, deep within the relativistic region. Only very close to the ISCO do the curves diverge, with the higher values of $E_{\mathrm{SR}}$ and $J_{\mathrm{SR}}$ for more highly spinning black holes coming in nearly equal parts from the higher fluxes at fixed radii and the decreasing value of $r_{\text {ISCO }}$ in the lower bound of the integrals in Eqs. (9).

Using this refined model for test-particle mergers, we can recalculate how the dimensionless black-hole spin $\chi$ and mass $M$ evolve after the black hole has accreted a finite mass $\Delta M$ of test particles gravitationally inspiraling inwards from large radii. Following Thorne 7 , we find that this evolution is governed by the coupled first-order differential equations

$$
\begin{aligned}
\frac{d \chi}{d \Delta M} & =\frac{1}{M^{2}} \frac{d J}{d \Delta M}-\frac{2 \chi}{M} \frac{d M}{d \Delta M} \\
\frac{d M}{d \Delta M} & =E_{\mathrm{ISCO}}(\chi)-E_{\mathrm{SR}}\left(\chi, r_{\mathrm{ISCO}}(\chi)\right) \\
\frac{d J}{d \Delta M} & =M\left[L_{\mathrm{ISCO}}(\chi)-J_{\mathrm{SR}}\left(\chi, r_{\mathrm{ISCO}}(\chi)\right)\right] .
\end{aligned}
$$

In Fig. 3, we see how the spin $\chi$ evolves for different values of the initial spin $\chi_{0}$ (where the curves intersect the y axis $\left.\Delta M / M_{0}=0\right)$. The black curves show the predictions of our model, while the blue curves show those of Bardeen [4] without the scattering of gravitational waves by the black hole. As expected, in our model black holes with initial spins $\chi \leq \chi_{\lim }$ can only be spun up to this limiting value no matter how much mass they accrete. 


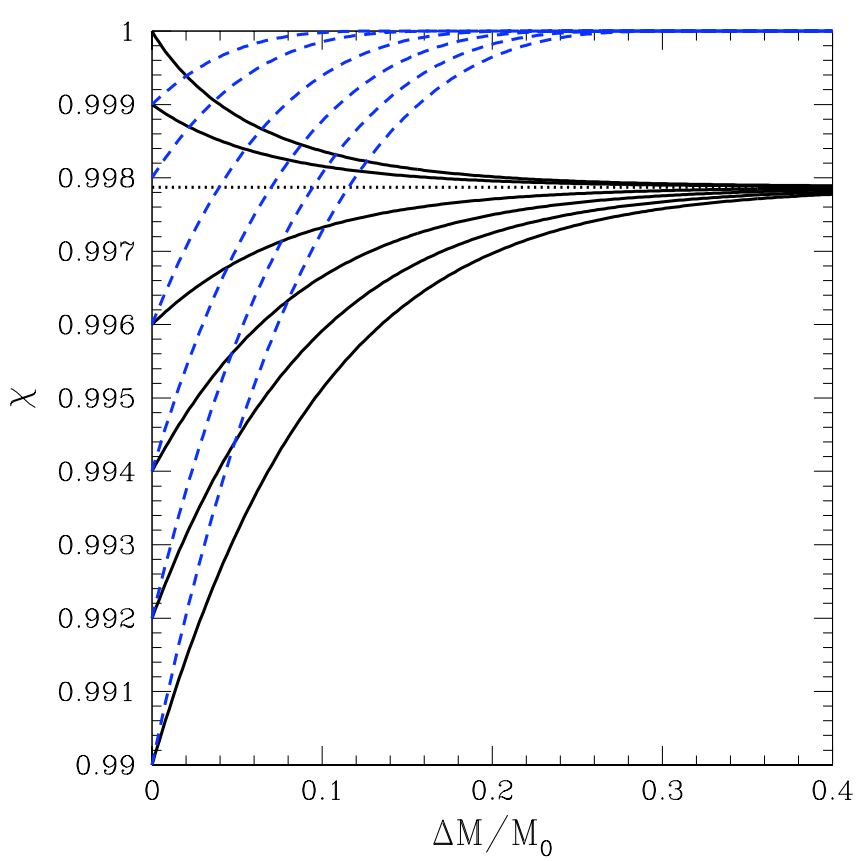

FIG. 3: The spin $\chi$ of a black hole initially of mass $M_{0}$ after binary mergers with a mass $\Delta M$ of test particles on quasicircular equatorial orbits. The black (solid) curves show the predictions of our model for different initial spins, while the blue (dashed) curves show the predicted spins in the absence of scattering. Spins in our model asymptote to $\chi_{\lim } \simeq 0.9979$ as shown by the horizontal dotted line, while without scattering black holes can spin all the way up to the Kerr limit.

More highly spinning black holes are spun down to near this limit after accreting $\gtrsim 10 \%$ of their initial mass. By contrast, in the Bardeen model black holes reach the Kerr limit $\chi=1$ after accreting a finite mass $\Delta M$ of test particles. For initially nonspinning holes, this mass is

$$
\Delta M=3 M_{0}\left(\sin ^{-1} \sqrt{2 / 3}-\sin ^{-1} 1 / 3\right) .
$$

Radiatively efficient black holes have a luminosity

$$
L=\varepsilon \frac{d \Delta M}{d t} c^{2}
$$

where $\varepsilon=1-E_{\mathrm{ISCO}}(\chi)$ is the radiative efficiency. In Fig. 4, we show the efficiency $\varepsilon$ of the black holes in Fig. 3 as a function of the total mass $\Delta M$ of test particles they have accreted. If the superradiant scattering of gravitational waves is neglected, the black holes spin up to the Kerr limit $\chi=1$ after which they are capable of converting a fraction $\varepsilon=1-1 / \sqrt{3}=0.423$ of accreted mass into radiant energy. By contrast, accounting for superradiant scattering reduces the maximum black-hole spin to $\chi_{\lim }=0.998$. Although this limiting spin is only $0.2 \%$ below the Kerr limit, the corresponding limiting radiative efficiency $\varepsilon_{\lim }=1-E_{\mathrm{ISCO}}\left(\chi_{\lim }\right)=0.320$ is $24 \%$ below that of a maximally spinning black hole. The inability of astrophysical black to radiate more efficiently

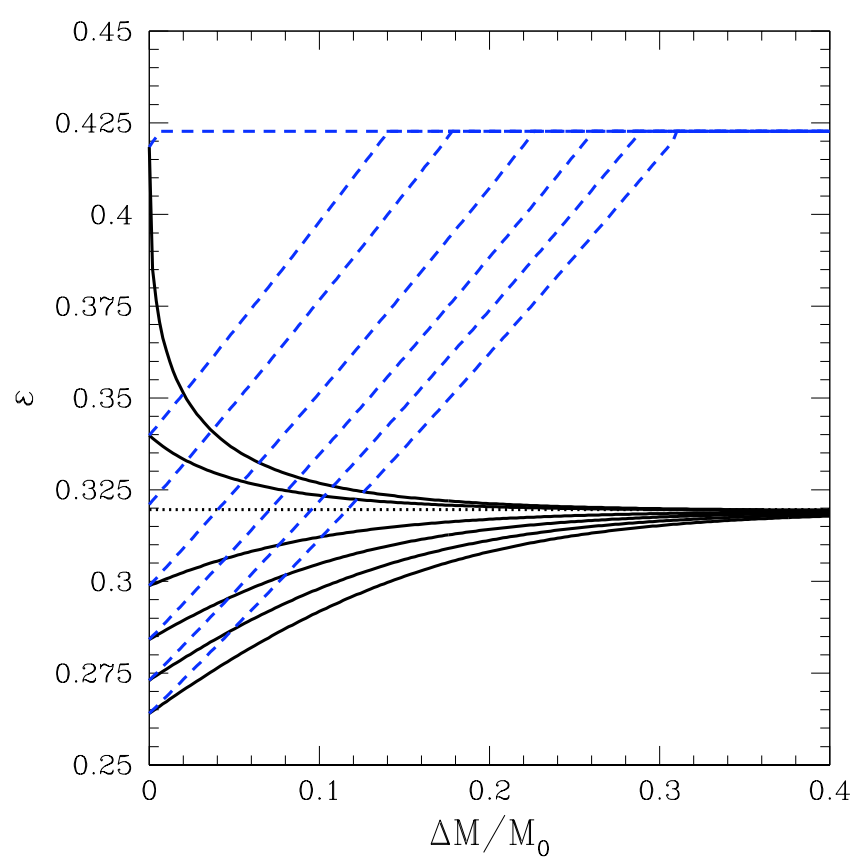

FIG. 4: The radiative efficiency $\varepsilon=1-E_{\mathrm{ISCO}}(\chi)$ of the accreting black holes shown in Fig. 3. In the Bardeen model (dashed blue curves), the black holes spin up to the Kerr limit $\chi=1$ after accreting a finite mass $\Delta M$. The radiative efficiency of a maximally spinning black hole is $\varepsilon=1-1 / \sqrt{3}=$ 0.423. In our model (solid black curves) which includes the superradiant scattering of gravitational waves, the black hole spins asymptotically approach $\chi_{\mathrm{lim}}$. A black hole with this spin has the substantially lower efficiency $\varepsilon_{\text {lim }}=0.320$.

than $\chi_{\text {lim }}$ will affect efforts to estimate black-hole masses from their luminosities, such as that by Soltan [30] to constrain the mass function of supermassive black holes from the quasar luminosity function.

\section{COMPARISON WITH NUMERICAL RELATIVITY}

How do the predictions of our model compare with those of other published models? In Fig. 5, we again show $\partial \chi_{f} / \partial q$, this time as a function of the initial spin $\chi_{i}$. The value of $\chi_{i}$ for which a given curve crosses the horizontal dotted line $\partial \chi_{f} / \partial q=0$ determines the maximum spin $\chi_{\lim }$ to which a black hole can be spun up by test-particle mergers. We see that the superradiant scattering of gravitational waves produced during the inspiral reduces $\chi_{\lim }$ from the Kerr limit as predicted by Bardeen 4, HB 23, and K 25] and shown by the blue (short-dashed) curve to $\chi_{i}=0.9979$ shown by the black (solid) curve. The red (long-dashed) curve shows the predictions of the BKL 24] model described in Sec. III. As discussed previously, this model artificially reduces $\chi_{\lim }$ to $\chi_{i}=0.948$ by neglecting the spin dependence of the 


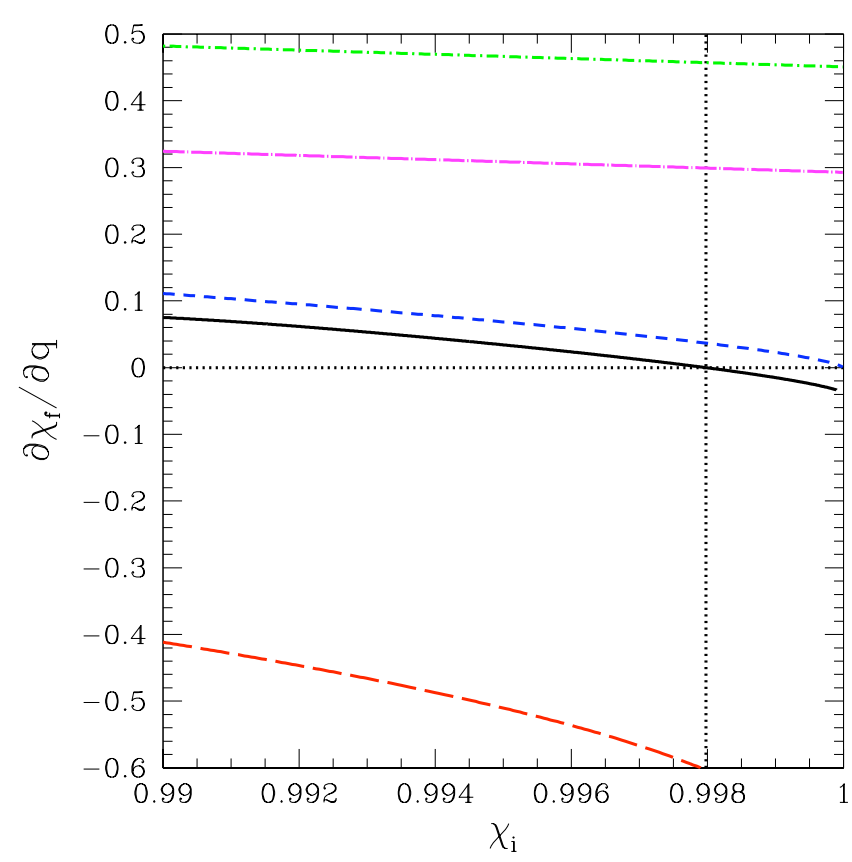

FIG. 5: The change in final spin per unit test-particle mass $\partial \chi_{f} / \partial q$ as a function of the initial spin $\chi_{i}$. The solid black curve shows the predictions of this paper, with spin down $\left(\partial \chi_{f} / \partial q \leq 0\right.$, horizontal dotted line) possible for spins $\chi_{i} \geq$ $\chi_{\lim }$ (vertical dotted line). The short-dashed blue curve shows how this result changes when superradiant scattering is neglected as in the models of Bardeen 4], HB [23, and K 25]. The long-dashed red curve gives the BKL 24] test-particle prediction, while the short dash-dotted green and long dashdotted magenta curves are the predictions of the AEI 31 and FAU [32] fitting formulas.

final mass. The green (short dash-dotted) and magenta (long dash-dotted) curves are the predictions of the AEI 31 and FAU 32 "fitting formulas" for the final spins.

The fitting-formula approach proposes a specific functional form for the dependence of the given quantity on the parameters $\left\{q, \chi_{1}, \chi_{2}\right\}$ and a small number of constant coefficients. These coefficients are adjusted until the fitting formula best reproduces the results of a sample of numerical simulations. Once the fitting formula has been calibrated in this manner, it should be able to predict the results of any future simulations. The AEI and FAU fitting formulas were calibrated with an extensive sample of numerical simulations of binary mergers with varying mass ratios and spins, none of which produced a black hole whose final spin exceeded the Kerr limit. Yet Fig. 5 shows that both curves have $\partial \chi_{f} / \partial q>0$ even for $\chi_{i}=1$, implying that test-particle mergers can spin black holes above the Kerr limit. One possible explanation of this unphysical prediction is that the fitted coefficients were misdetermined because of the limited range of mass ratios in the sample of simulations with which they were calibrated. A second explanation is that the proposed

\begin{tabular}{|c||c|c|c|}
\hline \multicolumn{1}{|c||}{} & \multicolumn{3}{c|}{ Initial spins $\chi_{i}$} \\
\hline$\chi_{i}$ & $-0.43757[13]$ & $0[12]$ & $1[15]$ \\
\hline \hline \multicolumn{1}{|c||}{} & \multicolumn{3}{c|}{ Final spins $\chi_{f}$} \\
\hline $\mathrm{NR}$ & $0.547812 \pm 0.000009$ & $0.68646 \pm 0.00004$ & $0.951 \pm 0.004$ \\
\hline $\mathrm{KLP}$ & 0.520861 & 0.686354 & 0.996439 \\
\hline $\mathrm{KLP}^{\prime}$ & 0.509269 & 0.674197 & 0.986947 \\
\hline $\mathrm{K}[\mathbf{2 5}]$ & 0.521153 & 0.687036 & 0.998805 \\
\hline BKL [24] & 0.505148 & 0.663086 & 0.959107 \\
\hline $\mathrm{AEI}[31]$ & 0.546646 & 0.686460 & 0.961491 \\
\hline FAU [32] & 0.548602 & 0.6860 & 0.9540 \\
\hline BK [33] & 0.547562 & 0.6893 & 0.9504 \\
\hline \hline \multicolumn{1}{|c||}{} & \multicolumn{3}{c|}{ Final masses $M_{f} / M$} \\
\hline $\mathrm{NR}$ & $0.961109 \pm 0.000003$ & $0.95162 \pm 0.00002$ & $\ldots$ \\
\hline KLP & 0.979028 & 0.974530 & 0.920918 \\
\hline KLP ${ }^{\prime}$ & 0.979268 & 0.974984 & 0.932995 \\
\hline K [25] & 0.979039 & 0.974565 & 0.916181 \\
\hline FAU [32] & 0.962877 & 0.9515 & 0.9255 \\
\hline BK [33] & 0.964034 & 0.9530 & 0.9009 \\
\hline
\end{tabular}

TABLE I: A comparison between the final spins and masses determined by numerical simulations and those predicted by various fitting formulas. All three simulations begin with equal-mass binaries whose spins are aligned or antialigned with the orbital angular momentum and have magnitudes given in the first row. These simulations are described more fully in the references provided. The next 8 rows give the final spin $\chi_{f}$ as determined by NR or predicted in the referenced papers. KLP are the predictions of this paper. The final 6 rows give the corresponding predictions for the final mass $M_{f} / M$.

functional forms are inadequate to describe the massratio or spin dependence of the final spin for any choice of coefficients. Further numerical simulations, particularly at smaller mass ratios, are needed to determine which of these explanations is correct. This problem is not just restricted to test-particle mergers; the AEI and FAU formulas predict final spins above the Kerr limit for mass ratios as large as $q=0.283$ and $q=0.2434$, respectively.

Although the AEI and FAU fitting formulas break down in the test-particle limit, by design they agree closely with simulations of comparable-mass mergers like those with which they were calibrated. In Table I] we compare our model, extrapolated to equal-mass mergers according to Eqs. (11), with both the fitting formulas and the most accurate numerical simulations of equalmass $(q=1)$, equal-spin $\left(\chi_{1}=\chi_{2}=\chi_{i}\right)$, aligned mergers. Both the nonspinning $\left(\chi_{i}=0\right)[12$ and antialigned $\left(\chi_{i}=-0.43757\right)$ [13 simulations were performed with the spectral-methods code developed by the CaltechCornell group. The $\chi_{i}=1$ numerical result [15] listed in the third column is an extrapolation to maximal spins of a series of simulations produced with the BAM finitedifference code used by the Jena and FAU groups.

The first row gives the calculated values of the final spin $\chi_{f}$ for these three numerical-relativity (NR) simu- 
lations. The next 7 rows give the final spins predicted for these binaries by many different published formulas. The second row lists the predictions of this paper, Kesden-Lockhart-Phinney (KLP). Our prediction for the nonspinning merger agrees with this numerical result to about $10^{-4}$. An agreement this good between a testparticle extrapolation and an equal-mass simulation can only be a coincidence, as is demonstrated by the third row $\mathrm{KLP}^{\prime}$. Here we have substituted $S_{f}^{\prime}$ from Eq. 12 for $S_{f}$ from Eq. 11b] in our prediction $\chi_{f}=S_{f} / M_{f}^{2}$. Although $S_{f}^{\prime}$ has the same symmetries and limiting behavior as $S_{f}$, the prediction changes by about $1 \%$ when extrapolating all the way to equal masses. Agreement beyond this accuracy must be considered coincidental unless we discover a fundamental reason to prefer $S_{f}$ over $S_{f}^{\prime}$.

The fourth row lists the predictions of Kesden [25, which was the starting point for this paper but neglected the superradiant scattering of downward-going radiation. Comparing the rows labeled KLP and K, we see that this scattering only reduces the final spin by about $0.25 \%$ even for binaries initially spinning at the Kerr limit. Although this effect seems negligible, it is potentially detectable since several gravitational-wave observables depend very sensitively on $\chi$ near the Kerr limit. We shall elaborate on this in the final discussion in Sec VI. Rows 5 through 8 list the predictions of other published formulas. We have already discussed the BKL model adequately; its close agreement with the extrapolation to maximally spinning binaries is a fortuitous coincidence. The AEI, FAU, and BK 33 fitting formulas all do excellent jobs of reproducing the numerical results, though the exact agreement of the AEI formula with the nonspinning simulation results from this simulation being included in the set with which this formula was calibrated.

The final 6 rows of Table 1 show how our predicted final masses $M_{f}$, extrapolated from the test-particle limit as before, compare with the numerical simulations and numerically calibrated fitting formulas. Our predictions overestimate $M_{f}$ compared to the simulations and fittingformula predictions for the antialigned and nonspinning mergers. This may result from our failure to account for the energy carried away by gravitational-wave emission after the merger. While this emission becomes negligible compared to that during the inspiral as $q \rightarrow 0$, significant emission after the formation of a common horizon can occur for equal-mass mergers. We plan to incorporate radiation during the plunge and ringdown stages of the merger in future work. The final mass has not been reliably determined for the maximally spinning case, which perhaps accounts for the greater discrepancy between the different fitting formulas. These formulas included only a limited number of highly spinning mergers in the set with which they were calibrated.

It is interesting to note when comparing rows $\mathrm{K}$ and KLP that superradiant scattering actually increases the predicted final mass for the maximally spinning merger, unlike in the other two cases. This is counterintuitive, since the top panel of Fig. 2 indicates that superradi- ant scattering extracts the most energy $E_{\mathrm{SR}}\left(\chi, r_{\mathrm{ISCO}}\right)$ from the most highly spinning black holes. However, as the test particle inspirals, superradiant scattering reduces the spin $\chi$, which moves the ISCO radius $r_{\text {ISCO }}(\chi)$ outwards and increases the ISCO energy $E_{\mathrm{ISCO}}(\chi)$. For large spins, the steep increase in $E_{\mathrm{ISCO}}(\chi)$ with spin more than compensates for the additional energy $E_{\mathrm{SR}}\left(\chi, r_{\mathrm{ISCO}}\right)$ extracted, thus increasing the final mass $M_{f}$.

Until numerical relativists can accurately simulate mergers with high spins and small mass ratios in an acceptable amount of time, we will need to rely on a combination of fitting formulas and test-particle extrapolations. These two methods complement each other, and any model that attempts to make predictions throughout the entire region $0 \leq q \leq 1$ should take advantage of both approaches. The fitting formulas are most accurate predicting the result of mergers close in parameter space to the simulations with which they were calibrated. Currently this consists mostly of comparable-mass mergers with $q \geq 0.1$. The test-particle extrapolations like the one proposed in this paper are most reliable for $q \ll 1$. We can readily modify the AEI formula 31] for aligned mergers

$$
\chi_{f}=\tilde{\chi}+\tilde{\chi} \eta\left(s_{4} \tilde{\chi}+s_{5} \eta+t_{0}\right)+\eta\left(2 \sqrt{3}+t_{2} \eta+t_{3} \eta^{2}\right),
$$

with

$$
\tilde{\chi} \equiv \frac{\chi_{1}+q^{2} \chi_{2}}{1+q^{2}},
$$

to incorporate the results of this paper. The first step in this modification was already taken in Rezzolla et al. [34, where it was recognized that setting the coefficient of the term proportional to $\eta$ equal to $2 \sqrt{3}$ would reproduce the test-particle prediction for nonspinning black holes in the absence of superradiant scattering of gravitational waves. We propose that all terms linearly proportional to $\eta$ can be replaced by our result for $\partial \chi_{f} / \partial q$ from Sec. IV

$$
\chi_{\mathrm{KLP}}=\tilde{\chi}+\frac{\partial \chi_{f}}{\partial q}(\tilde{\chi}) \eta+\left(t_{2}+s_{5} \tilde{\chi}\right) \eta^{2}+t_{3} \eta^{3} .
$$

This eliminates two of the coefficients from Eq. (17) and guarantees that the formula behaves properly in the testparticle limit. The highly accurate equal-mass nonspinning simulation [12] can still be used to determine $t_{2}$ as in Barausse and Rezzolla [31] if desired. If the formula no longer has enough degrees of freedom to reproduce existing numerical results, additional terms proportional to highers powers of $\eta$ and $\tilde{\chi}$ can be added. These new terms will not affect the test-particle behavior, as $\eta \rightarrow 0$ in this limit, but can help to fit comparable-mass mergers where $\eta \lesssim 0.25$. There also remains additional freedom in our choice of $\tilde{\chi}$. Although the choice given in Eq. (18) possesses the desired symmetry and limiting behavior

$$
\begin{aligned}
q \rightarrow 0 & \Rightarrow \tilde{\chi} \rightarrow \chi_{1} \\
q \rightarrow \infty & \Rightarrow \tilde{\chi} \rightarrow \chi_{2},
\end{aligned}
$$


this choice is not unique and alternatives may prove more suitable. An iterative approach for determining $\tilde{\chi}$ as a function of $\left\{q, \chi_{1}, \chi_{2}\right\}$ as in BKL [24] and Kesden 25] should be explored as well.

This same approach can be used to improve fitting formulas for the final mass. Tichy and Marronetti 32 . proposed the fitting formula

$$
\frac{M_{f}}{M}=1+4\left(m^{0}-1\right) \eta+16 m^{a 1} \eta^{2}\left(\chi_{1}+\chi_{2}\right),
$$

where $m^{0}$ and $m^{a 1}$ are fitting coefficients. In the testparticle limit $\eta \rightarrow q \rightarrow 0$ this reduces to

$$
\frac{\partial}{\partial \eta}\left(\frac{M_{f}}{M}\right)=4\left(m^{0}-1\right)=-0.194 \text {. }
$$

Our Eq. 11a in this limit predicts

$$
\frac{\partial}{\partial \eta}\left(\frac{M_{\mathrm{KLP}}}{M}\right)=E_{\mathrm{ISCO}}\left(\chi_{1}\right)-E_{\mathrm{SR}}\left(\chi_{1}, r_{\mathrm{ISCO}}\right)-1,
$$

correctly capturing the spin dependence of the binding energy $E_{\mathrm{ISCO}}$ and extracted energy $E_{\mathrm{SR}}$ on the larger black hole's spin. We can use this result to replace the term linear in $\eta$ in Eq. 21) to yield

$$
\frac{M_{\mathrm{KLP}}}{M}=1+\frac{\partial}{\partial \eta}\left(\frac{M_{\mathrm{KLP}}}{M}\right)(\tilde{\chi}) \eta+32 m^{a 1} \eta^{2} \tilde{\chi} .
$$

Notice that we have also replaced the sum of the spins in the third term with $\tilde{\chi}$, as the sum did not possess the required limiting behavior of Eq. 20). Additional terms proportional to higher powers in $\eta$ can be added to improve the agreement with comparable-mass simulations without affecting the test-particle behavior.

\section{DISCUSSION}

The superradiant scattering of gravitational waves emitted during the inspiral sets a fundamental upper limit $\chi_{\lim }=0.9979 \pm 0.0001$ on the spin a black hole may attain by accreting test particles on quasicircular equatorial orbits. For this limiting spin, the energy and angular momentum advected when the test particle falls from the ISCO combines with that extracted by superradiant scattering to leave the spin $\chi=S / M^{2}$ of the black hole unchanged. Black-hole spins greater than $\chi_{\text {lim }}$ will be reduced by mergers with test particles on quasicircular equatorial orbits, even though the physical angular momentum $S$ and mass $M$ will both increase.

Jacobson and Sotiriou 35] recently argued that small but finite-mass particles on highly hyperbolic orbits could spin black holes above $\chi_{\lim }$ and even above the Kerr limit itself. However, this study neglected gravitational radiation and any superradiant enhancement, which could be considerable for these highly spinning mergers. Such hyperbolic orbits are also extremely unlikely in any realistic astrophysical scenario.
Our limit is nearly indistinguishable from the limit $\chi_{\text {gas }}=0.9980 \pm 0.0002$ for black holes grown through thin-disk gas accretion [7, suggesting that spin measurements alone cannot determine the relative contribution of these two channels of black-hole growth. If gas accretion occurs through an advection-dominated accretion flow (ADAF) rather than a thin disk, the limiting blackhole spin is reduced to $\chi_{\mathrm{ADAF}} \simeq 0.96$, and the launching of jets can further reduce this spin to $\chi_{\text {jets }} \simeq 0.93$ [11]. Since our limit $\chi_{\mathrm{lim}}$ is larger than for other modes of black-hole growth, it seems to be a fairly robust upper bound on spins that can be obtained by any astrophysical means.

Although SBHs are grown primarily through gas accretion rather than test-particle mergers, our limiting spin $\chi_{\lim }$ can still be astrophysically relevant. Gas accretion occurs episodically, when galactic major mergers produce global torques that funnel large amounts of gas to galactic centers [36, 37. Accretion of this gas onto the SBH leads to AGN feedback [38] which simulations demonstrate is capable of suppressing further accretion and black-hole growth [39]. By contrast, compact objects such as white dwarfs, neutron stars, and stellar-mass black holes (effectively test particles when orbiting an SBH) will inspiral continuously as they are scattered into the "loss cone" of orbits for which gravitational inspiral occurs more rapidly than subsequent scattering [40. Although the same global torques driven by galactic major mergers may enhance the rate of these extreme-mass-ratio inspirals (EMRIs), other dynamical processes will refill the loss cone even between periods of AGN activity [41. Event rates for EMRIs depend sensitively on highly uncertain factors such as the dynamical state, star-formation history, binary fraction, and initial mass function within nuclear star clusters. The capture rate for $10 M_{\odot}$ black holes by a $10^{6} M_{\odot} \mathrm{SBH}$ can be as high as $10^{-4} \mathrm{yr}^{-1}$ immediately following a nuclear starburst, and could average $10^{-6} \mathrm{yr}^{-1}$ for nucleated spiral galaxies like the Milky Way [42. If such a galaxy has not had a major merger since redshift $z \simeq 1$ (about 7.5 Gyr ago), its SBH would aqcuire a fraction

$$
\frac{\Delta M}{M_{0}} \simeq \frac{10 M_{\odot} \times 10^{-6} \mathrm{yr}^{-1} \times 7.5 \mathrm{Gyr}}{10^{6} M_{\odot}}=0.075
$$

of its mass through EMRIs. We see from Fig. 3 that this is a large enough fraction to drive the SBH spin towards our limiting value $\chi_{\text {lim }}$ from the initial spins $\chi_{0} \gtrsim 0.9$ expected from gas accretion. This suggests that EMRIs can potentially affect SBH spin evolution, though modest gas accretion can also occur between major mergers and not all EMRIs will be on circular, equatorial orbits.

Even if our limiting spin $\chi_{\text {lim }}$ can be realized astrophysically, does its marginal difference from the Kerr limit have any observable consequences? The binding energy per unit mass at the ISCO $1-E_{\mathrm{ISCO}}$ depends very sensitively on $\chi$ near the Kerr limit, and sets an upper bound on the AGN efficiency $\varepsilon \equiv L / \dot{M} c^{2}$, where $L$ is the AGN luminosity, $\dot{M}$ is the accretion rate, and $c$ is the speed of 
light. Soltan 30] argued that the total cosmological mass in SBHs could be estimated by equating the observed energy emitted by AGN to that released during theoretical models of SBH growth. If spins at the Kerr limit were used for an analysis of this kind instead of our limiting spin $\chi_{\text {lim }}=0.9979$, the AGN efficiency $\varepsilon$ would be overestimated by $E_{\mathrm{ISCO}}\left(\chi_{\mathrm{lim}}\right)-E_{\mathrm{ISCO}}(1)=0.1031$ leading to a corresponding underestimate in the total SBH mass.

In addition to their influence on the SBH spin, the EMRIs themselves are an important source for gravitationalwave detectors such as LIGO and LISA. Decreasing the spin from the Kerr limit to $\chi_{\lim }$ moves the ISCO radius out from $r_{\mathrm{ISCO}}(1)=m_{1}$ to $r_{\mathrm{ISCO}}\left(\chi_{\mathrm{lim}}\right)=1.242 m_{1}$, and decreases the ISCO orbital frequency from $\Omega_{\mathrm{ISCO}}(1)=$ $0.50 m_{1}^{-1}$ to $\Omega_{\mathrm{ISCO}}\left(\chi_{\lim }\right)=0.42 m_{1}^{-1}$. This decrease could be observable in high signal-to-noise EMRIs seen by both these experiments. Our limiting spin $\chi_{\lim }$ is thus not only an interesting consequence of general relativity, but also one that has potentially observable implications for astrophysics and gravitational-wave detection.

Acknowledgements. We thank Scott Hughes for generously allowing us to use a version of his GREMLIN (Gravitational Radiation in the Extreme Mass ratio LIMit) code to calculate the energy and angular momentum radiated during the inspiral. We also thank Andrew Benson, Chris Hirata, Pedro Marronetti, Uli Sperhake, and Kip Thorne for useful conversations. The authors acknowledge support from NASA BEFS Grant No. NNX07AH06G (PI:Phinney). M. Kesden also acknowledges support from NSF Grant No. PHY-0601459 (PI: Thorne).
[1] R. P. Kerr, Phys. Rev. Lett. 11, 237 (1963).

[2] C. S. Reynolds, A. J. Young, M. C. Begelman and A. C. Fabian, Astrophys. J. 514, 164 (1999).

[3] L. W. Brenneman and C. S. Reynolds, Astrophys. J. 652, 1028 (2006).

[4] J. M. Bardeen, Nature 226, 64 (1970).

[5] N. I. Shakura and R. A. Sunyaev, Astron. Astrophys. 24, 337 (1973).

[6] B. B. Godfrey, Phys. Rev. D 1, 2721 (1970).

[7] K. S. Thorne, Astrophys. J. 191, 507 (1974).

[8] R. Popham and C. F. Gammie, Astrophys. J. 504, 419 (1998) arXiv:astro-ph/9802321.

[9] S. A. Balbus and J. F. Hawley, Astrophys. J. 376, 214 (1991).

[10] C. F. Gammie, S. L. Shapiro and J. C. McKinney, Astrophys. J. 602, 312 (2004) arXiv:astro-ph/0310886.

[11] A. J. Benson and A. Babul, Mon. Not. Roy. Astron. Soc. 397, 1302 (2009) arXiv:0905.2378 [astro-ph.HE]].

[12] M. A. Scheel, M. Boyle, T. Chu, L. E. Kidder, K. D. Matthews and H. P. Pfeiffer, Phys. Rev. D 79, 024003 (2009) arXiv:0810.1767 [gr-qc]].

[13] T. Chu, H. P. Pfeiffer and M. A. Scheel, arXiv:0909.1313 [gr-qc].

[14] G. Lovelace, R. Owen, H. P. Pfeiffer and T. Chu, Phys. Rev. D 78, 084017 (2008) arXiv:0805.4192 [gr-qc]].

[15] P. Marronetti, W. Tichy, B. Brugmann, J. Gonzalez and U. Sperhake, Phys. Rev. D 77, 064010 (2008).

[16] C. O. Lousto, Y. Zlochower, arXiv:1009.0292 [gr-qc]].

[17] J. A. Gonzalez, U. Sperhake and B. Brugmann, Phys. Rev. D 79, 124006 (2009) arXiv:0811.3952 [gr-qc]].

[18] C. O. Lousto, H. Nakano, Y. Zlochower et al., Phys. Rev. Lett. 104, 211101 (2010). arXiv:1001.2316 [gr-qc]].

[19] C. O. Lousto, H. Nakano, Y. Zlochower et al., arXiv:1008.4360 [gr-qc]].

[20] P. C. Peters and J. Mathews, Phys. Rev. 131, 435 (1963).

[21] S. A. Teukolsky and W. H. Press, Astrophys. J. 193, 443 (1974).

[22] L. S. Finn and K. S. Thorne, Phys. Rev. D 62, 124021 (2000) arXiv:gr-qc/0007074.

[23] S. A. Hughes and R. D. Blandford, Astrophys. J. 585, L101 (2003).
[24] A. Buonanno, L. E. Kidder and L. Lehner, Phys. Rev. D 77, 026004 (2008).

[25] M. Kesden, Phys. Rev. D 78, 084030 (2008) arXiv:0807.3043 [astro-ph]].

[26] E. Newman and R. Penrose, J. Math. Phys. 3, 566 (1962).

[27] S. A. Teukolsky, Astrophys. J. 185, 635 (1973).

[28] M. Sasaki and T. Nakamura, Prog. Theor. Phys. 67, 1788 (1982).

[29] S. A. Hughes, Phys. Rev. D 61, 084004 (2000) [Erratumibid. D 63, 049902 (2001 ERRAT,D65,069902.2002 ERRAT,D67,089901.2003)] arXiv:gr-qc/9910091.

[30] A. Soltan, Mon. Not. Roy. Astron. Soc. 200, 114 (1982).

[31] E. Barausse and L. Rezzolla, arXiv:0904.2577 [gr-qc].

[32] W. Tichy and P. Marronetti, Phys. Rev. D 78, 081501 (2008) arXiv:0807.2985 [gr-qc]].

[33] L. Boyle and M. Kesden, Phys. Rev. D 78, 024017 (2008) arXiv:0712.2819 [astro-ph]].

[34] L. Rezzolla, P. Diener, E. N. Dorband, D. Pollney, C. Reisswig, E. Schnetter and J. Seiler, Astrophys. J. 674, L29 (2008) arXiv:0710.3345 [gr-qc]].

[35] T. Jacobson, T. P. Sotiriou, Phys. Rev. Lett. 103, 141101 (2009). arXiv:0907.4146 [gr-qc]].

[36] J. C. Mihos and L. Hernquist, Astrophys. J. 464, 641 (1996) arXiv:astro-ph/9512099.

[37] J. E. Barnes and L. Hernquist, Astrophys. J. 471, 115 (1996).

[38] J. Silk and M. J. Rees, Astron. Astrophys. 331, L1 (1998) arXiv:astro-ph/9801013.

[39] T. Di Matteo, V. Springel and L. Hernquist, Nature 433, 604 (2005) arXiv:astro-ph/0502199.

[40] J. Frank and M. J. a. Rees, Mon. Not. Roy. Astron. Soc. 176, 633 (1976).

[41] S. Sigurdsson, Class. Quant. Grav. 20, S45 (2003) arXiv:astro-ph/0304251.

[42] S. Sigurdsson and M. J. Rees, Mon. Not. Roy. Astron. Soc. 284, 318 (1997) arXiv:astro-ph/9608093.

[43] Throughout this paper we express physical quantities in units where Newton's constant $G$ and the speed of light $c$ are unity, in which case the spin angular momentum $S$ has dimensions of $M^{2}$. 